Document downloaded from:

http://hdl.handle.net/10251/165836

This paper must be cited as:

Araque Monrós, MC.; Gil-Santos, L.; Monleón Pradas, M.; Más Estellés, J. (2020). New bioreactor for mechanical stimulation of cultured tendon-like constructs: design and validation. Expert Review of Medical Devices. 17(10):1115-1121. https://doi.org/10.1080/17434440.2020.1825072

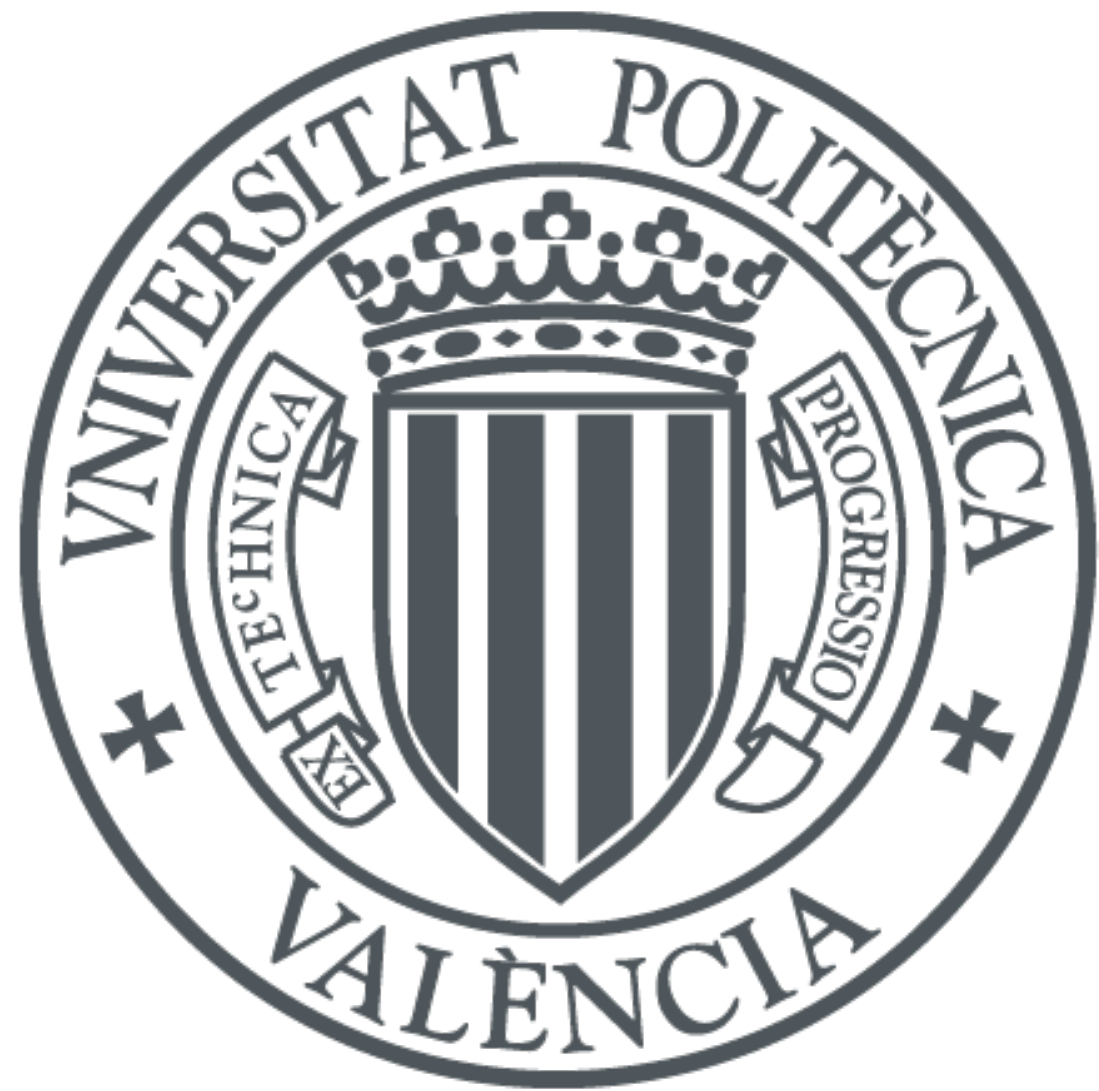

The final publication is available at

https://doi.org/10.1080/17434440.2020.1825072

Copyright Taylor \& Francis

Additional Information 


\section{New bioreactor for mechanical stimulation of cultured tendon-like constructs. Design and validation.}

Araque-Monrós M.C. ${ }^{1,2,4}$, Gil-Santos, L. ${ }^{2,3}$, Monleón Pradas M. ${ }^{1,2}$, Más-Estellés J. ${ }^{2^{*}}$

${ }^{1}$ CIBER en Bioingeniería, Biomateriales y Nanomedicina, Valencia, Spain.

${ }^{2}$ Centro de Biomateriales e Ingeniería Tisular, Universitat Politécnica de València, 46022 Valencia, Spain.

${ }^{3}$ Instituto Universitario de Investigación en Enfermedades Músculo-esqueléticas

Universidad Católica de Valencia (San Vicente Mártir).C/Quevedo, 2, 46001, Valencia, Spain.

${ }^{4}$ Current address: AIMPLAS, Plastics Technology Centre, Gustave Eiffel, 4, 46980 Paterna; Valencia, Spain.

*Corresponding author

Tel: 0034963877007 ext. 75271

Fax: 0034963877276

E-mail: jmas@fis.upv.es 


\section{Abstract}

Objective: Although several different types of bioreactor are currently available with mechanical stimulation of constructs or prostheses for tendon regeneration, they are in many cases expensive and difficult to operate. This paper proposes a simple bioreactor to mechanically stimulate up to three constructs for tendon and ligament repair, composed of a stainless-steel frame and an electric motor.

Methods: The deformation is produced by a cam wheel, whose eccentricity defines the maximum deformation. The test samples, braids of PLA seeded in surface with mouse fibroblasts, are immersed in the culture medium during mechanical stimulation.

Results: Its advantages over existing similar bioreactor designs include: easy renewal of the culture medium and an external electric motor to avoid heating and contamination issues.

After 14 days of stretching, the culture samples showed enhanced cellular proliferation and cell fibre alignment in addition to higher production of type I collagen. The cells initially seeded on the braid surface migrated to the inside of the braid.

Conclusion: Although the results obtained have a poor statistical basis, they do suggest that the bioreactor could be usefully applied to stimulate constructs for tendon and ligament repair. Anyway, further experiments should be conducted in future.

Keywords: bioreactor; mechanical stimulation; construct; regeneration; tendon. 


\section{Introduction}

Prostheses have been used to restore damaged tendons and ligaments over the past century. These tissues can be replaced either by natural fibres (silk, catgut or grafts (autografts, allografts or xenografts) and synthetic materials (nylon, polyethylene, silastic, Teflon, PLA, PGA, etc.) [1-4], although this solution presents certain long-term problems, like loosening of the bone anchorage, slackness of the prosthesis, or stiffness of the member $[5,6]$. Regenerating the native tissue with implants focuses on Tissue Engineering techniques, based on a combination of cellular cultures and biomaterials [7$11]$.

The mechanical conditions under which this regeneration process occurs are especially important. It has been repeatedly shown that regeneration of this tissue is more effective when the cells are mechanically stimulated, as in the body's natural reaction to an injury. Cell growth on non-stressed supports leads to deficiently organized tissues, while the application of a mechanical force during regeneration improves the properties of the regenerated tissue [12-18], mainly due to the collagen fibres of stressed tissues being aligned in the direction of the force applied and the increased production of type I collagen.

In vitro experiments should resemble as much as possible the in vivo conditions of the biological processes during regeneration (acidity, the medium surrounding the tissue, nearby tissues, mechanical stress, anchorage to muscle and bone, etc.). Bioreactors aim to achieve similar ambient conditions for the cells. Cell cultures should be carried out in controlled conditions in a sterile environment, as regards temperature, load ranges and deformation, etc. [19]. Bioreactors should also be able to deal with several samples at once. 
During mechanical stimulation, stresses are transferred to the cells through the biomaterial support on which they are seeded. Different types of bioreactors can be used for different types of tissues [18, 20-29]. The aim of this work was to design, build and validate an easily operated bioreactor with an external motor that could be used for tissue engineering techniques to mechanically stimulate cell-biomaterial constructs for tendon and ligament regeneration. This ex vivo partial tendon and ligament tissue regeneration would be an advantage during subsequent surgical implantations, leading to better integration of the prostheses in the host body.

\section{Materials and methods}

The bioreactor is made up of a stainless steel frame, capable of being sterilized before each experiment, either in an autoclave or sanitized with ethanol solution. One end of the sample is anchored to the fixed part of the frame and the other to the moving part, which is oscillated by an adjustable cam wheel (its eccentricity defines the maximum deformation) to subject the sample to variable stretching cycles. To avoid the contaminating the cells under culture, the motor is outside the incubator and transmits the movement by a steel cable inside a sheath. The culture medium is placed in a Petri dish, so that the samples subjected to stretching cycles remain immersed in the culture medium, which can be renewed as frequently as required by simply replacing the Petri dish.

The motor can be placed as far from the incubator as needed to avoid heat transfer or contamination. The appropriate temperature and ambient conditions can be set up inside the incubator.

\subsection{Bioreactor. Frame design.}


The $180 \times 85 \times 55 \mathrm{~mm}^{3}, 304$ medical grade stainless steel frame was supplied by Mecanizados, S.A. (Alfafar, Valencia, Spain). The glass tubes were manufactured by VidraFoc (Valencia, Spain). Figure 1 shows a side view, plan and photo of the bioreactor.

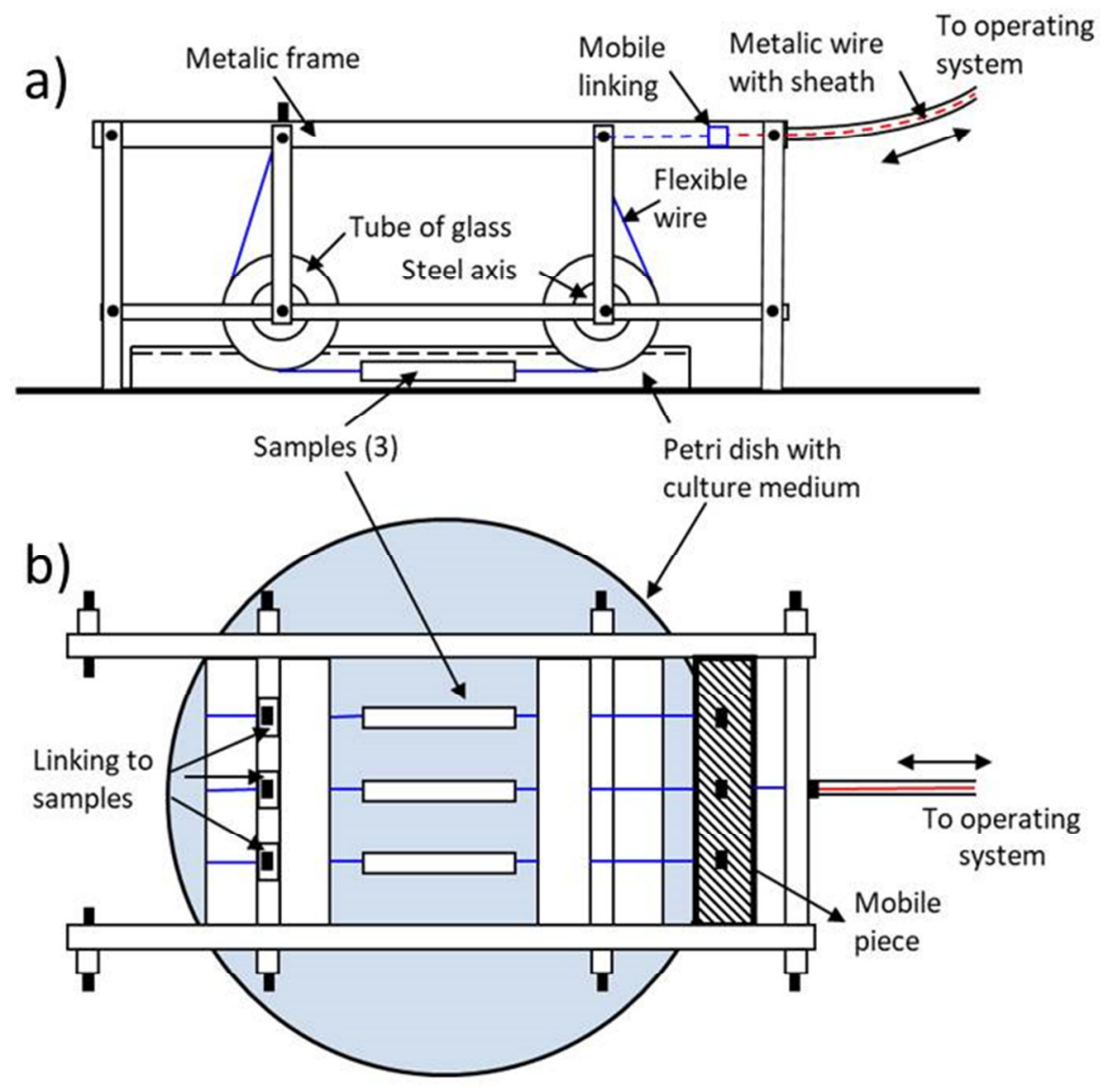

c)

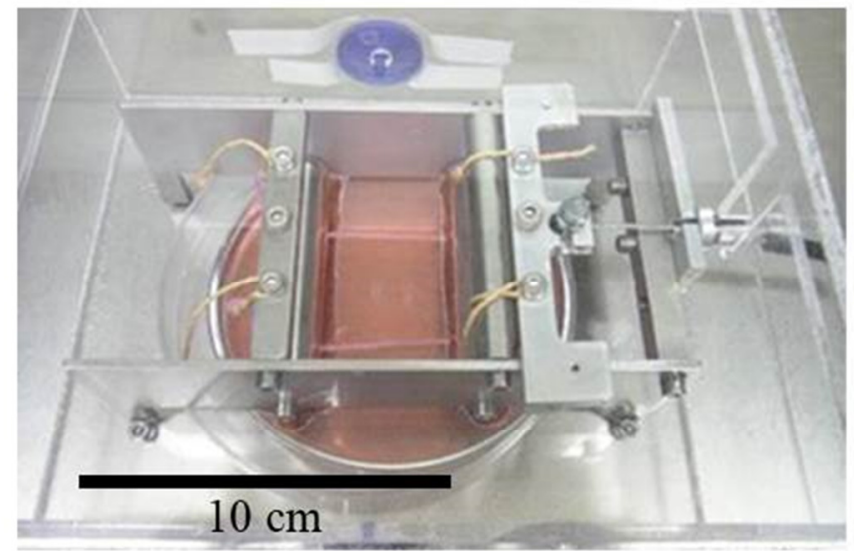


Fig. 1 a) Bioreactor side view, b) plan and c) photo. The two samples at the left end are oscillated by the moving part on the right. Petri dish containing the culture medium can also be seen.

The samples are tied to two cables with very high rigidity anchored to the frame in such a way that practically all the deformation is absorbed by the sample. The moving part of the frame is oscillated by an external motor and applies tensile forces to the samples. The frequency of stretching cycles can be controlled by adjusting the motor speed. To avoid metal parts coming in contact with the culture medium, the steel cables slide over two glass tubes. Two steel rods (10 mm outer diameter) pass through the glass tubes (20 $\mathrm{mm}$ outer and $10 \mathrm{~mm}$ inner diameter) to support the forces on the samples and avoid breaking the glass.

The samples can be of any length, although only the part immersed in the culture medium can be seeded with cells. The samples remain immersed in the Petri dish containing the culture medium. Three samples can be tested simultaneously.

A methacrylate box $\left(220 \times 180 \times 80 \mathrm{~mm}^{3}\right)$ was designed to minimize the risk of sample contamination and facilitate the transport of the bioreactor from the laminar flow chamber (where the samples are attached to the frame) to the incubator chamber.

\subsection{Bioreactor. Power system.}

The samples are tensed at one end by a unit containing a $12 \mathrm{~V}$ (maximum voltage) DC electric motor and a gearbox (gear ratio r=104) Beru A350 (BorgWarner Inc. Auburn Hills, (WHQ) MI, USA), as shown in Figure 2. 


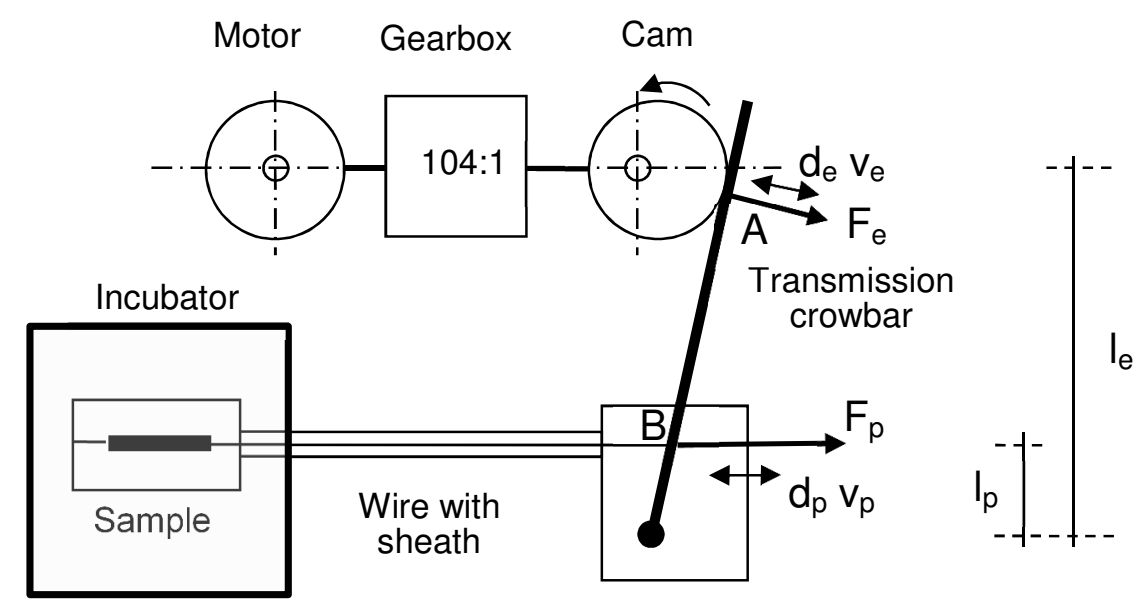

Fig. 2 Bioreactor power system

The cam wheel is turned by a shaft from the gearbox, that in turn moved a bar (contact point A). Such bar, at point B, moved the wire connecting the mobile piece of frame of bioreactor. Therefore, depending on the amplitude of movement of cam $\left(d_{e}\right)$, on the lengths $l_{e}$ and $l_{p}$, on the intensity consumed by the motor $(I)$, on the necessary intensity to overcome every mechanical friction $\left(I_{0}\right)$, and on constant of motor $k^{\prime}$ (relationship between torque and current, $k^{\prime}=0.0155 \mathrm{~T} \cdot \mathrm{m}^{2}$ ), we can obtain the force applied to stretch the sample $\left(F_{p}\right)$ and the amplitude of the applied deformation $\left(d_{p}\right)$ :

$$
F_{p}=\frac{r \cdot k^{\prime}\left(I-I_{0}\right) \pi}{d_{e}} \frac{I_{e}}{I_{p}} \quad(1) \quad d_{p}=\frac{I_{p}}{l_{e}} d_{e}
$$

The motor is powered by an adjustable DC Gold Source Power Supply DF1731 SB 030 V, 0-3 A Dual Out (Apliquem Micrones 21, Valencia, Spain). The system only works in the displacement-control mode. The maximum sample displacement can be 
varied by installing a cam wheel with a different eccentricity. The frequency of the movement applied to the sample can be controlled by altering the applied voltage.

\subsection{Bioreactor calibration}

The initial deformation applied to the sample was lower than expected due to its sheath being too elastic. It was therefore necessary to calibrate the bioreactor by mounting a load cell U3 (HBM Ibérica, Madrid, Spain) with a range 0-200 N and a resolution of 0.1 $\mathrm{N}$, and a displacement sensor (a ruler with a resolution of $0.25 \mathrm{~mm}$ ) to measure the magnitudes really applied to the samples. These sensors were then disassembled and no real time acquisition was possible.

When the power supply was adjusted to $6 \mathrm{~V}$, the samples were subjected to a stretching movement of frequency $0.3 \mathrm{~Hz}$. The maximum load applied to the samples was $10 \mathrm{~N}$ and at an amplitude of $1 \mathrm{~mm}$, or $2 \%$ strain, according to the length of the tested sample (50 $\mathrm{mm})$. The strength and deformation values were between the usual values quoted in the literature $[15,17]$. As the motor can supply a maximum $12 \mathrm{~V}$ and the revolutions are directly related to the applied voltage, the frequency of the movement can reach up to 0.6 Hz. The width of movement only depends on the cam wheel eccentricity, while the force applied to the sample depends on the displacement and on the features of the sample. This force applied to the sample could vary during the test due to stress relaxation because of the polymeric nature of the sample or even to the extracellular matrix maturation process, but the strain will remain unchanged in both cases.

\subsection{Static and dynamic cell cultures}

Dynamic tests were carried out to verify the bioreactor's ability to mechanically stimulate the seeded samples and the effective transfer of the mechanical stimulus to the 
cells. Also static tests were carried out. Other aims were to verify that the bioreactor could be easily operated when the culture medium was changed and that the external motor avoided contamination and temperature rises. The samples tested were made up of hollow PLA braids seeded on the surface with mouse fibroblasts (cellular line L929 in passage 3). These braids have been self-manufactured and have been proposed as elements of a construct intended for tendon regeneration. The materials, manufacturing process and physicochemical properties can be found in $[8,30]$. Culture times were 7 (T7) and 14 (T14) days.

The $100 \mathrm{~mm}$ long PLA braid was tied at both ends to a $50 \mathrm{~mm}$ long Kevlar braid. The PLA sample was sanitized with EtOH $70 \%$ solution, conditioned overnight with PBS and DMEM with $4.5 \mathrm{~g} \cdot \mathrm{l}^{-1} \mathrm{D}$-glucose without FBS for $24 \mathrm{~h}$ before cell culture. The samples were then surface-seeded with $100 \mu$ l of a cellular suspension $\left(1 \times 10^{5}\right.$ cells).

The samples were incubated at $37^{\circ} \mathrm{C}$ and $5 \% \mathrm{CO}_{2}$ foralong $1 \mathrm{~h}$, mounted in the previously sterilized bioreactor, and covered with culture medium (approx. $110 \mathrm{ml}$ ) $(n=2)$. The set was carried to the incubator and connected to the motor. This connection is easily made by inserting the head of the wire into a hole on the moving part of the frame. The culture medium was renewed every three days by replacing the Petri dish under the frame of the bioreactor, and mechanical stimulus was applied $9 \mathrm{~h}$ per day, with frequency of $0.3 \mathrm{~Hz}$, maximum load $10 \mathrm{~N}$, and $2 \%$ maximum strain.

The braided material without mechanical stimulation was used as the control sample (static test) in a Petri dish in the same conditions as the dynamic tests (same cell type and equal time points).

\subsection{Analyses of cultured samples}


The static and dynamically cultured samples were then subjected to standard morphology and immunofluorescence tests [30].

\subsubsection{Morphology}

Scanning electronic microscopy (JEOL JSM-5410, Japan) was used to observe the surface morphology of the braided PLLA. The samples were washed twice in phosphate buffer solution (PBS) and fixed with a solution of glutaraldehyde $2.5 \%$ for $1 \mathrm{~h}$ at $4^{\circ} \mathrm{C}$, then washed 3-4 times with PBS at 5 min intervals. Prior to SEM observation, the samples were dehydrated by changing the water for EtOH. The samples were dried and gold sputtered for further analysis. Micrographs were taken at $10 \mathrm{kV}$.

The outer braid surface was studied for static and dynamic cell cultures at T7 and T14. To observe the internal braid morphology, the sample was cut lengthwise with a scalpel. The inner braid surface of the dynamic cultures was observed at T14.

\subsubsection{Immunofluorescence}

Cell distribution, morphology and type I collagen were examined by immunofluorescence analysis using a confocal microscope with inverted laser (Leica TCS SP2 AOBS, Germany).

The samples were washed with phosphate buffer solution (PB $0.1 \mathrm{M}$ ) for 5 minutes at room temperature. Blocking buffer was added and maintained for $2 \mathrm{~h}$, after which the primary and secondary antibodies (Col I:PB ratio 1:40 and Cy3:PB ratio 1:200) were successively added (10 $\mu$ l per sample of bodipy-FL phalloidin (Invitrogen) was also added to stain the cytoskeleton), incubated in the dark at room temperature and washed three times with PB $0.1 \mathrm{M}$ every 5 minutes at room temperature. The nuclei were then stained with a solution of DAPI-Vectashield (Vector Laboratories) 1:1000 (DAPI: $\mathrm{H}_{2} \mathrm{O}$ ) 
and incubated for 15-30 min in the dark. The solution was then removed and the samples washed three times every 5 minutes with PB $0.1 \mathrm{M}$ and mounted on microscope slides with a drop of medium.

Static and dynamic cell cultures were observed at T7. 


\section{Results and discussion}

After 7 and 14 days culture, the samples were fixed and the morphology of fibroblasts seeded on the braid surface was analysed by SEM. The main objective was not to analyse the changes in the samples, but to verify the bioreactor's performance and the effects of the mechanical stimulation.

The 7 and 14-day static and dynamic cell culture micrographs can be seen in Figure 3. The dynamic culture cell density is clearly higher than that of the static cell cultures for the same time (see figures bI, bII and bIII versus figures aI and aII for T7, and figures bIV, bV and bVI versus figures aIII and aIV for T14). The dynamically cultured cells can be seen to adhere to the entire braid surface. Increased cell density and better cell alignment in the direction of the fibres can also be seen with a more extended and fusiform morphology in the dynamic cultures. 
$\mathrm{T} 7$

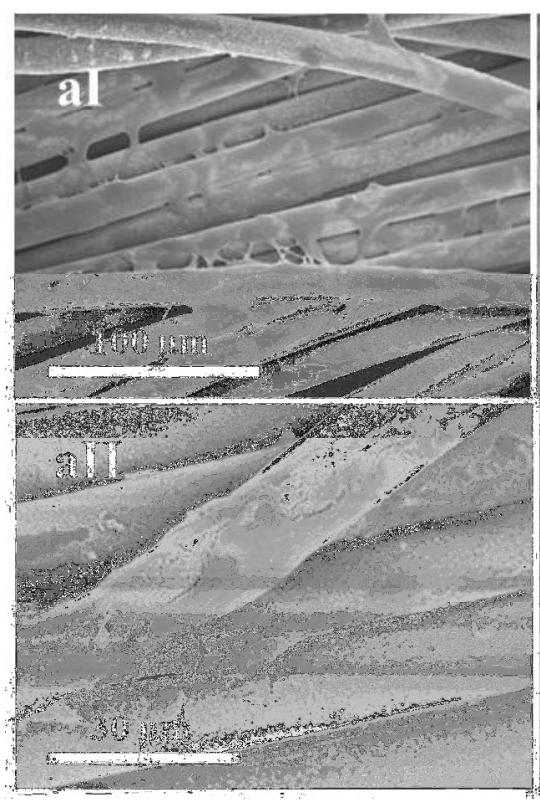

13:

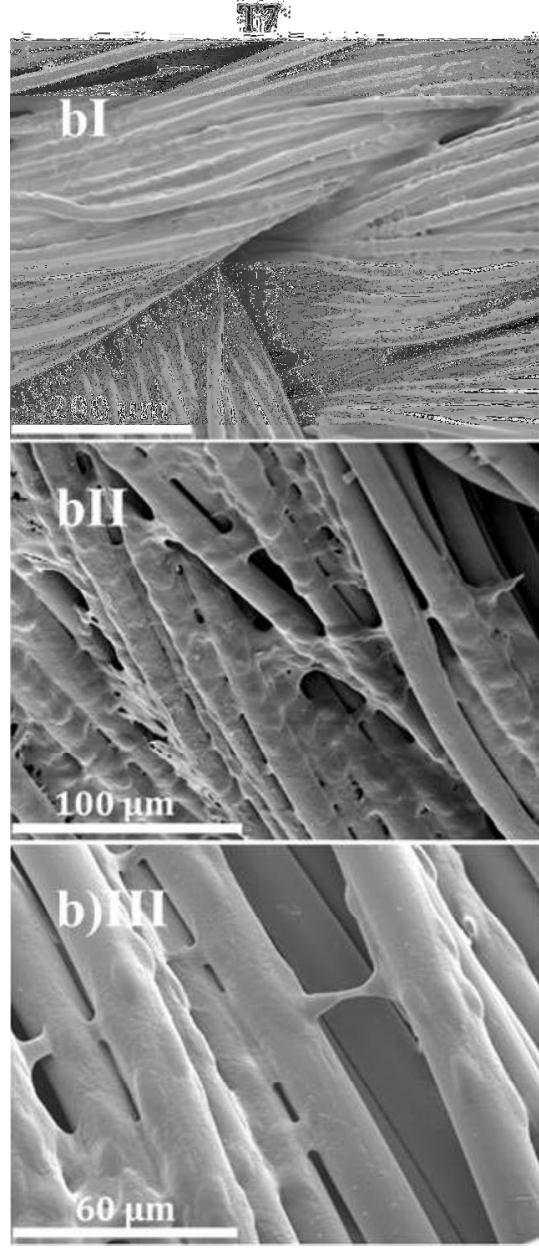

$\mathrm{T} 14$

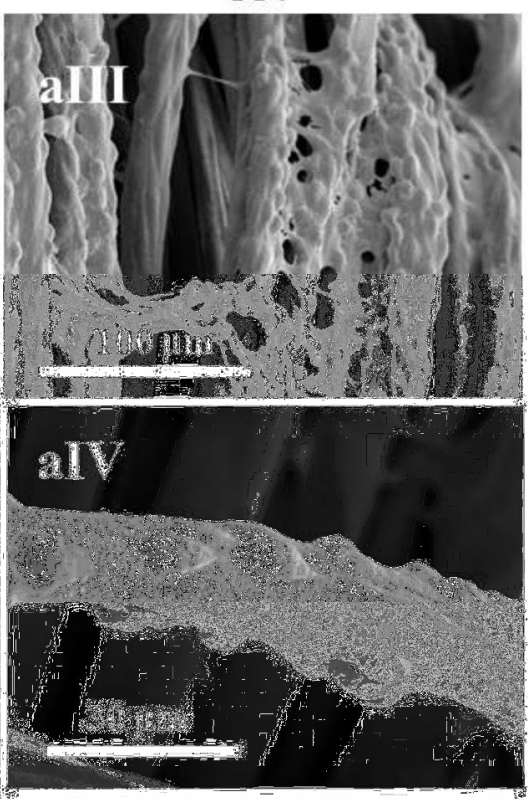

S11:

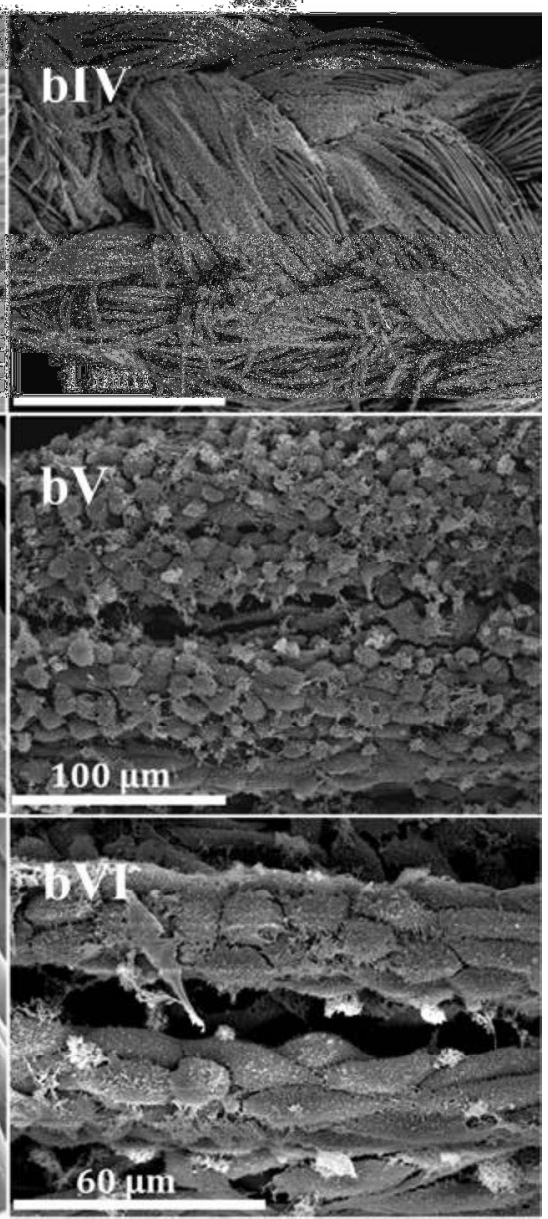

Fig. 3 SEM images of morphology of L929 cells on PLA braid surface after 7 and 14 days (T7 and T14) of static culture (set a) and dynamic culture (set b), with different 
magnifications: very low (bI and bIV), low (aI, aIII, bII and bV) and high (aII, aIV, bIII and bVI).

The inner part of the dynamically cultured braid was also analysed by SEM images (Figure $4 \mathrm{a}$ and $\mathrm{b}$ ), which revealed the presence of internal cells, thus suggesting that the cells had migrated from the surface. As happened outside the braid, the cells exhibit an extended morphology in the same direction as the microfibres.
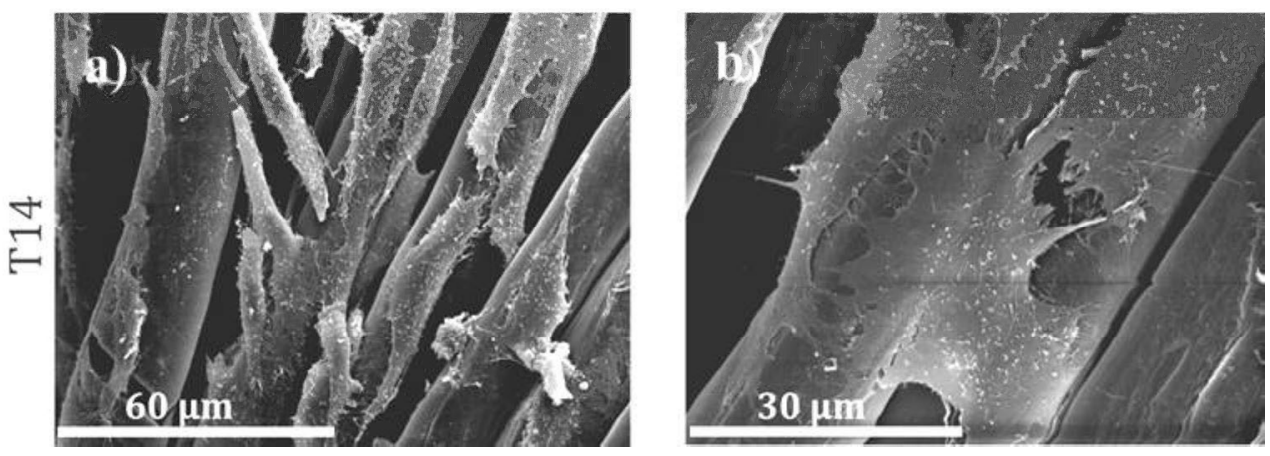

Fig. 4 SEM images of L929 fibroblasts morphology inside the PLA braid after 14 days of dynamic cell culture with two different magnifications: a) low and b) high.

Figure 5 shows immunofluorescence images after dyeing the cytoskeleton with actin and the nuclei with DAPI. The cells subjected to a mechanical stimulus seem to express more type I collagen than those cultured in static conditions (see figures bI, bII and bIII versus figures aI, aII and aIII), as reported by other authors [31, 32].

Type I collagen is the predominant protein of tendinous tissue and its expression and secretion are key factors in the regeneration process of this tissue $[33,34]$. In both cases, a well-developed actin cytoskeleton can be seen. 


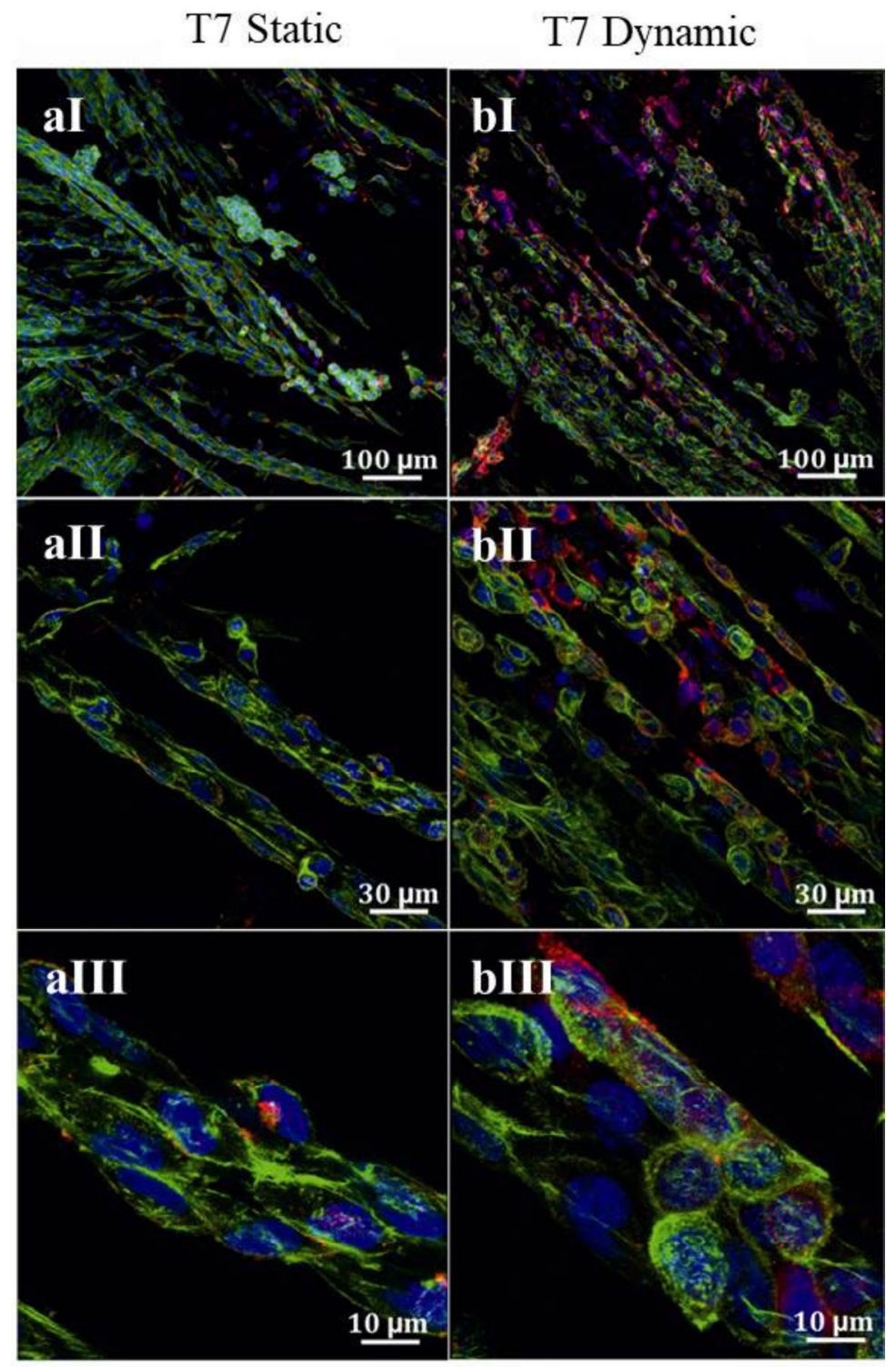

Fig. 5 Immunofluorescence images of type I collagen (red) and dyed actin cytoskeleton (green) of fibroblasts seeded on the PLA braid after 7 days of static (set a) and dynamic (set b) cultures with different magnifications: low (aI and bI), high (aII and bII) and very high (aIII and bIII). The cell nuclei were dyed with DAPI (blue).

Even though these results are only preliminary results, they show that the prototype bioreactor mechanically stimulated the tendon and ligament constructs, produced greater cellular proliferation, cell alignment in the direction of the fibres and cell migration to the inside of the sample. 
The cell alignment indicated that the stress applied to the fibres was transmitted to the cells and increased the quantity of type I collagen.

\section{Conclusions}

Even though our tests were not statistically significant because of their insufficient number, they do suggest that the proposed bioreactor mechanically stimulated the tendon and ligament constructs. The strain applied to the sample can be easily modified by simply changing the cam wheel. The bioreactor also has the important advantages over those currently available of being easy to operate and its easy renovation of the culture medium. Further experiments should be conducted in order to state the effectiveness of the bioreactor.

\section{Acknowledgments}

This work was carried out thanks to the financial support of AITEX (Alcoi, Alicante, Spain), through the research contract "Development of braided biomaterials for biomedical applications".

The authors thank John Wiley and Sons for the permission to reprint part of Figure 5.

\section{Conflicts of interest: None}

Funding: Researching contract "Development of braided materials for biomedical applications".

Ethical approval: Not required 


\section{References}

1. Murray GA, Semple JC. A review of work on artificial tendons. J Biomed Eng. 1979;1:177-184. doi:10.1016/0141-5425(79)90040-2

2. Ricci JL, Gona AG, Alexander H, Parsons JR. Morphological characteristics of tendon cells cultured on synthetic fibers. J Biomed Mater Res. 1984;18(9):1073-1087. DOI: $10.1002 / \mathrm{jbm} .820180910$

3. Hunter JM, Salisbury RE. Flexor-tendon reconstruction in severely damaged hands. A two-stage procedure using a silicone-dacron reinforced gliding prosthesis prior to tendon grafting. J Bone Joint Surg Am. 1971;53(5):829-858.

4. Hunter JM, Singer DI, Jaeger SH, Mackin EJ. Active tendon implants in flexor tendon reconstruction. J Hand Surg-Am. 1988;13:849-859.

5. Mohamed A, Oliva F, Nardoni S, Maffulli N. Failed synthetic graft after acute Achilles tendon repair. Muscles 2017;7(2):396-402. DOI: 10.11138/mltj/2017.7.2.396

6. Grace Walden, Xin Liao, Simon Donell, Mike J. Raxworthy, Graham P. Riley, Aram Saeed. A clinical, biological, and biomaterials perspective into tendon injuries and regeneration. Tissue Eng Pt B. 2017:23(1):44-58.

7. Araque Monrós C, Gil Santos L, Gironés Bernabé S, Más Estellés J, Monleón Pradas M. Universitat Politècnica de València. Procedimiento de obtención de una prótesis biodegradable. Patent of invention $n^{\circ} \mathrm{P} 201130919.2011$.

8**. Araque-Monrós María C, Gamboa-Martínez Tatiana C, Luis Gil Santos, Sagrario Gironés Bernabé, Manuel Monleón Pradas, Jorge Más Estellés. New concept for a regenerative and resorbable prosthesis for tendon and ligament: Physicochemical and biological characterization of PLA-braided biomaterial. J Biomed Mater Res A. 2013;101A:3228-3237. DOI: 10.1002/jbm.a.34633. This reference is of considerable importance because the manufacturing process and parameters of the braid are described here.

9*. Freeman Joseph W, Woods Mia D, Laurencin Cato T. Tissue engineering of the anterior cruciate ligament using a braid-twist scaffold design. J Biomech. 2007;40:2029-2036. This reference is important because it is one of the first attempts to manufacture a braided prosthesis for tendon and ligament.

10. Laurencin Cato T, Freeman Joseph W. Ligament tissue engineering: An evolutionary materials science approach. Biomaterials. 2005;26:7530-7536.

11. Merolli A, Joyce TJ. Biomaterials in Hand Surgery. Milan: Springer; 2009.

12. Moreau Jodie E, Bramono Diah S, Horan Rebecca L, Kaplan David L, Altman Gregory H. Sequential Biochemical and Mechanical Stimulation in the Development of Tissue-Engineered Ligaments. Tissue Eng Pt A. 2008:14(7):1161-1172.

13. Nirmalanandhan Victor S, Rao Marepalli, Shearn Jason T, Juncosa-Melvin Natalia, Gooch Cindi, Butler David L. Effect of scaffold material, construct length and mechanical stimulation on the in vitro stiffness of the engineered tendon construct. $\mathrm{J}$ Biomech. 2008;41(4):822-828. DOI:10.1016/j.jbiomech. 2007.11.009

14. Ruwan D. Sumanasinghe, Jason A. Osborne, Elizabeth G. Loboa. Mesenchymal stem cell-seeded collagen matrices for bone repair: Effects of cyclic tensile strain, cell density, and media conditions on matrix contraction in vitro. J Biomed Mater Res A. 2009;88(3):778-786. 
15. Saber S, Zhang AY, Ki SH, Lindsey DP, Smith RL, Riboh J, et al. Flexor tendon tissue engineering: bioreactor cyclic strain increases construct strength. Tissue Eng Pt A. 2010;16(6):2085-2090.

16. Tohyama H, Yasuda K. The effects of stress enhancement on the extracellular matrix and fibroblasts in the patellar tendon. J Biomech. 2000;33(5):559-565.

17. Wang T, Lin Z, Day RE, Gardiner B, Landao-Bassonga E, Rubenson J, et al. Programmable mechanical stimulation influences tendon homeostasis in a bioreactor system. Biotechnol Bioeng. 2013;110:1495-1507. doi:10.1002/bit.24809

18. Youngstrom DW, Barrett JG. Engineering Tendon: Scaffolds, Bioreactors, and Models of Regeneration. Stem Cells Int. 2016;Article ID 3919030, 11 pages. doi:10.1155/2016/3919030

19*. Wang T, Gardiner BS, Lin Z, Rubenson J, Kirk TB, Wang A, et al. Bioreactor design for tendon/ligament engineering. Tissue Eng Pt B-Reviews. 2013;19(2):133-146. doi: 10.1089/ten.teb.2012.0295. This reference is of importance because the key components, design preferences and required criteria of a bioreactor for tendon and ligament are identified.

20. Abousleiman Rita I, Reyes Yuliana, McFetridge Peter, Sikavitsas Vassilios. Tendon Tissue Engineering Using Cell-Seeded Umbilical Veins Cultured in a Mechanical Stimulator. Tissue Eng Pt A. 2009;15(4):787-795.

21. Masuda Taisuke, Takahashi Ichiro, Anada Takahisa, Arai Fumihito, Fukuda Toshio, Takano-Yamamoto Teruko, et al. Development of a cell culture system loading cyclic mechanical strain to chondrogenic cells. J Biotechnol. 2008;133(2):231-238.

22. Xu ZC, Zhang WJ, Li H, Cui L, Cen L, Zhou GD, et al. Engineering of an elastic large muscular vessel wall with pulsatile stimulation in bioreactor. Biomaterials. 2008;29(10):1464-1472.

23. TC-3F Ebers Medical Technology, S.L. http://www.ebersmedical.com/ Accesed May $15^{\text {th }} 2019$.

24. CellScale biomaterials testing. https://cellscale.com/ Accesed March $16^{\text {th }} 2020$.

25. Wei Lee Lim, Ling Ling Liau, Min Hwei Ng, Shiplu Roy Chowdhury, Jia Xian Law. Current Progress in Tendon and Ligament Tissue Engineering. Tissue Eng Regen Med. 2019:16:549-571

26. Alberto Sensini, Luca Cristofolini. Biofabrication of Electrospun Scaffolds for the Regeneration of Tendons and Ligaments. Materials. 2018:11:1963(43 pp) doi:10.3390/ma11101963

27. Ramin Oftadeh, Brianne K. Connizzo, Hadi Tavakoli Nia, Christine Ortiz, Alan J. Grodzinsky. Biological connective tissues exhibit viscoelastic and poroelastic behavior at different frequency regimes: Application to tendon and skin biophysics. Acta Biomaterialia. 2018:70:249-259.

28. Mahshid Vashaghian, Chantal M. Diedrich, Behrouz Zandieh-Doulabi, Arie Werner, Theodoor H. Smit, J.P. Roovers. Gentle cyclic straining of human fibroblasts on electrospun scaffolds enhances their regenerative potential. Acta Biomaterialia. 2019:84:159-168.

29. Florian Helms, Skadi Lau, Melanie Klingenberg, Thomas Aper, Axel Haverich, Mathias Wilhelmi, et al. Complete Myogenic Differentiation of Adipogenic Stem Cells 
Requires Both Biochemical and Mechanical Stimulation. Annals of Biomedical Engineering. 2020:48:913-926.

30. Araque-Monrós, M.C., García-Cruz, D.M., Escobar-Ivirico, J.L., Gil-Santos, L., Monleón-Pradas, M., Más-Estellés, J. Regenerative and resorbable PLA/HA hybrid construct for tendon/ligament tissue engineering. Ann Biomed Eng. 2020; 48(2):757767.

31. Yang G, Crawford RC, Wang JHC. Proliferation and collagen production of human patellar tendon fibroblasts in response to cyclic uniaxial stretching in serum-free conditions. J Biomech. 2004;37(10):1543-1550.

32. Surrao DC, Fan JC, Waldman SD, Amsden BG. A crimp-like microarchitecture improves tissue production in fibrous ligament scaffolds in response to mechanical stimuli. Acta Biomater. 2012;8(10):3704-3713.

33. Wang, JH. Mechanobiology of tendon. J Biomech 2006:39(9):1563-1582.

34*. Chuanxin Zhang, Jun Zhu, Yiqin Zhou, et al. Tendon stem/progenitor cells and their interactions with extracellular matrix and mechanical loading, Stem Cells Int. 2019:Oct 13 doi:10.1155/2019/3674647. This reference is of importance because it is a review of the interactions between the mechanical loading and the pathological conditions of tendons. 